\title{
A Numerical Evaluation of an Infill Sampling Criterion in Artificial Neural Network-Based Optimization
}

\author{
Han Li, Leonardo Gutierrez, Masakazu Kobayashi, Osamu Kuwazuru, Hiroyuki Toda, and Rafael
} Batres

\begin{abstract}
Surrogate models can be used to replace expensive computer simulations for the purposes of optimization. In this paper, we propose an optimization approach based on artificial neural network (ANN) surrogate models and infill sampling criteria (ISC) strategy to evaluate design variables. The criterion for infill sample selection is a function which aims at identify design that offer potential improvement. We employ four widely used analytical benchmark problems to test the proposed approach. Our results show that a more accurate surrogate model obtained with fewer points is obtained when one includes the infill sample criterion to an ANN-based optimization.
\end{abstract}

Index Terms-Surrogate model, design variables, artificial neural network, infill sampling criteria, optimization, benchmark function.

\section{INTRODUCTION}

Optimization methods for black-box systems have applications in many engineering-domains. Most engineering design problems require high fidelity simulations to evaluate design variables. These simulations are based on mathematical models of some system of interest. Examples include finite element analysis (FEA) method for structural engineering problems or Navier-Stokes models in computational fluid dynamics (CFD). However, for many real world problems, despite steady advances in computing power, a single simulation can take many minutes, hours, or even days to complete. As a result, design optimization becomes impractical since it may require thousands or even millions of simulation evaluations. For example, in order to find the optimal material parameters, the finite element analysis (FEA) along with nanoindentation test is undertaken [1]-[3].

Therefore, to overcome this problem cheap approximating models (often termed "surrogate models") are sought. These are based on a limited number of calls to the high fidelity model. Once constructed, the surrogate model can replace the original high fidelity model for the purposes of optimization. Polynomial regression [4]-[6], radial basis function (RBF) [7], and Kriging [8], [9] are among some of the most

Manuscript received November 5, 2013; revised January 9, 2014.

Han Li, Leonardo Gutierrez, Rafael Batres, and Masakazu Kobayashi are with the Department of Mechanical Engineering, Toyohashi University of Technology, Toyohashi, Aichi, Japan (e-mail: \{lihan, Leonardo\}@ise.me.tut.ac.jp; m-kobayashi@me.tut.ac.jp; rbp@tut.jp).

Osamu Kuwazuru is with the Department Nuclear Power \& Energy Safety Engineering, University of Fukui, Fukui 910-8507, Japan (e-mail: kuwa@u-fukui.ac.jp).

Hiroyuki Toda is with the Department of Mechanical Engineering, Kyushu University, Kyushu, Japan (e-mail: toda@mech.kyushu-u.ac.jp). prominent and commonly used techniques. In this paper we concentrate on artificial neural network (ANN) surrogate models. ANN models have been successfully applied to many engineering problems. ANNs are universal approximates which have been mathematically proven to be able to approximate any continuous nonlinear function arbitrarily well over a compact interval to any degree of accuracy as long as they contain at least one hidden layer.

During an ANN-based optimization, the global optimum will not be found if we only utilize the ANN models built from a small set of initially sampled data, since the models are not globally accurate. Therefore, several new inputs should be added so that the ANN models can be updated, where each new input incorporates with all prior information. This process is performed by the infill sampling criterion (ISC) function. ISC function can adaptively select better additional sampling point to improve the surrogate model and find the optimum value at every iteration. This step is repeated until a time limit, evaluation budget, convergence, or model accuracy is reached.

Since the iterative strategy represents the heart of the surrogate-based optimization process, the choice of ISC is then of great importance. In [10], the expected improvement (EI) is introduced. Also, Viana [11] used the probability of improvement (PI) to update a Kriging surrogate model.

In this paper, we propose a criterion for infill sample selection that helps to select the points in the design space with the biggest contribution to the current error. In other words, it considers both the spatial position of the design variables and the areas of high estimated approximation error generated by the surrogate model. This criterion is simple and easy to use.

The rest of this paper is organized as follows. Section II introduces the setting of this problem. The proposed methodology is presented in Section III. Section IV we show some results from our approach and in the final section conclusions are drawn and areas of further research highlighted.

\section{Problem Setting}

We consider the system of interest as a black box that provides no information other than the measurements of system performance. In a typical approximation model the relationship between responses and design variables on a $k$-dimensional domain $D$ is expressed as

$$
y=f(x, u),
$$

where $y$ is the observed response, $\boldsymbol{x}$ is a vector of $k$ 
independent design variables whose values are unknown, and $u$ is a vector of design parameters whose values are fixed as a part of the problem specifications. In the case of a high fidelity model (such as FEA), the design parameters are assigned values, that is the response y must be a function $f_{L}$ of the parameters $\boldsymbol{x}$ and $u$ :

$$
y=f_{L}(\boldsymbol{x}, u)
$$

Assuming that we can afford to run the high fidelity analysis $N$ times, we sample for $N$ designs denoted by $\left(\left(\boldsymbol{x}^{(\mathbf{1})}, u\right),\left(\boldsymbol{x}^{(2)}, u\right), \ldots,\left(\boldsymbol{x}^{(N)}, u\right)\right)$, at which we obtain the responses $\left(y^{(1)}, y^{(2)}, \ldots, y^{(N)}\right)$. The surrogate model is built on these designs and responses.

The surrogate-based optimization problem is formulated as:

$$
\begin{gathered}
\operatorname{Min} E_{u}|f(\boldsymbol{x}, u)-z(\boldsymbol{x}, u)|, \\
\text { s.t. } x \in D \subseteq X^{k}
\end{gathered}
$$

where $E_{u}$ denotes the expectation with respect to $\mathrm{u}, f(x, u)$ is the experiment response, $z(x, u)$ is the prediction obtained by ANN model, and $D$ is the design space.

When the probabilistic distribution function $\varphi(u)$ is given, $E_{u}$ is calculated by the following equation:

$$
E_{u}=\int \varphi(u)|f(\boldsymbol{x}, u)-z(\boldsymbol{x}, u)| d u
$$

On the other hand, when the uncertain parameters deviate randomly within a certain interval, or the probabilistic distribution function is not given explicitly, the above computation is substituted by the average over m samples. In this case, a large number of samples can increase the accuracy of such a computation:

$$
E_{u}=\sum_{i=1}^{m} \mid\left(f\left(\boldsymbol{x}, u_{i}\right)-z\left(\boldsymbol{x}, u_{i}\right) \mid\right.
$$

\section{Methodology}

In this section, the methodological foundations of our approach are introduced. The flowchart for the optimization method based on ANN with ISC is shown in Fig. 1. The first step is to generate an initial data set based on some design of experiments. A surrogate model is then built based on true simulations from this initial sample. The second stage extracts knowledge from the surrogate to find points for model refinement referred to as updating. These update points are selected via an infill sampling criterion.

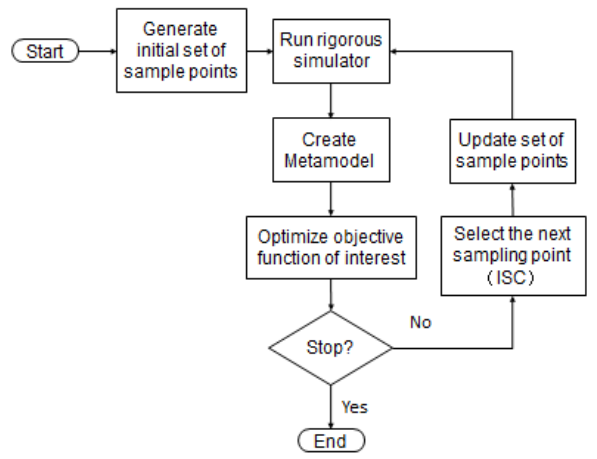

Fig. 1. Flowchart for ANN with ISC.

\section{A. Sampling Plan}

The first step consists of generating an initial population of design points either by random generation or by means of design of experiments (DOE) techniques [12]. DOE is the preferred sampling plan as it permits to evenly fill the design space with a limited number of points.

The most frequently used techniques in this stage of the process are latin hypercube sampling algorithm (LHS) [13], full-factorial design, orthogonal arrays and box-behnken design. Each of these methods has its own advantages and disadvantages depending on the characteristics of the design problem. Here, we use LHS design optimized with respect to the maximum criterion, which is common choice in many cases. The advantage of this method is that they divide uniformly the design space for each parameter and guarantee to have good space-filling properties.

Then, high fidelity model is executed for all the values of the input variables in the DOE specified in the previous step.

\section{B. Building a Surrogate}

After selecting the initial sample points an appropriate experimental design and performing the necessary computer runs, the next step is to build surrogate model. Surrogate model is the key to surrogate-based design optimization.

There are several approaches for building such models, here we choose to work with ANNs on the grounds that their training is inexpensive, yet, as we will see, they are sufficiently accurate for optimization purposes.

ANNs are computational models inspired by animal central nervous systems (in particular the brain) that are capable of machine learning and pattern recognition. ANNs can be seen as systems of interconnected "neurons" that can compute values from inputs by feeding information through the network. Three training methods are commonly used, namely back propagation, conjugate gradient, and Levenberg-Marquardt methods.

In Fig. 2, a sketch of a hierarchical neural network is shown. An ANN is a multilayered construction made up of one or more hidden layers placed between the input and output layers. The layers include several processing units called neurons. All of them are connected with variable weights that have to be determined.

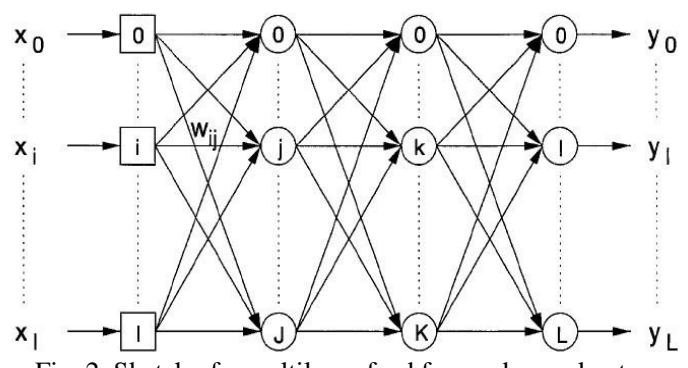

Fig. 2. Sketch of a multilayer feed forward neural net.

The inputs are operated and transformed into the output by the state transition rule as

$$
\begin{gathered}
v_{j}=\sum w_{i, j} y_{i}+\theta_{j}, \\
y_{j}=f\left(v_{j}\right),
\end{gathered}
$$

where $y_{i}$ in "(3)" and $y_{j}$ in "(4)" denote the output from a 
neuron $i$ acting as an input on neuron $j$ and the output of neuron $j$ respectively. $w_{i, j}$ is the synaptic weight, $\theta_{j}$ is the bias, and $v_{j}$ is the state variable of the synaptic weights which imply the connection strength between the neurons. A neuron in the network produces its output $y_{j}$ by processing the net input through an activation (transfer) function $f$. In this work, the activation function is given by the smooth sigmoidal function

$$
f\left(v_{j}\right)=1 /\left\{1+\exp \left(-v_{j}\right)\right\}
$$

taking values between 0 and 1 .

An ANN is trained by repeatedly presenting a series of input and output pattern sets to the network. The neural network gradually "learns" the relationship of interest by modifying the weights between its neurons to minimize the error between the actual and predicted output patterns of the training set. Then, a separate set of data called the test set to monitor network's performance. During training, the learning rule is used to iteratively adjust the weights and biases of the network in order to move the network outputs closer to the target values by minimizing the network performance indicator.

\section{Infill Sampling Criterion}

The cornerstone of any optimization strategy based on surrogate models is the choice of the updating method, i.e. given an initial global model, how to select the next sites where the expensive objective will be sampled. Perhaps the most obvious strategy is to re-sample in areas that appear promising in terms of the objective function value which predicted by the surrogate model. The success of this approach depends on the quality of the initial approximation. If the initial approximation is accurate, it is likely to lead the designer quickly to the global minimum or at least to a very good solution.

A second approach is to search areas of high estimated approximation error, i.e. in our case, to choose the design that maximizes the estimated error of the ANN predictor. This infill criterion uses information of the current model in order to assess the utility of evaluating this design on the actual problem. The infill criteria are used to increase the accuracy of the prediction by creating globally accurate surrogate models.

The offset (error) value $I_{z}(p)$ is as a quality index, which is the sum of the difference between the actual response and simulation data. In order to calculate this, we make a sampling, more precisely,

$$
I_{z}(p)=\sqrt{\frac{1}{m} \sum_{i=1}^{m}\left(z\left(u_{i}, p\right)-f\left(u_{i}, p\right)\right)^{2}},
$$

here, $f\left(u_{i}\right)$ is the actual response at points $u_{i}$, the function $z$ is a ANN model trained, and $\mathrm{m}$ is the number of experiment data related to the fix parameter $u$.

We try to select points with the biggest contribution to the current error. In order to estimate the representativeness of selected point $p$, we give a weight function, that is

$$
w_{i, j}=d\left(p_{i}, p_{j}\right)
$$

where $d\left(p_{i}, p_{j}\right)=1-\exp \left(-\left\|p_{i}-p_{j}\right\|^{2}\right)$, and $p_{j}$ is the original LHS data. This weight function gives the similarity of the design variables. When the two design variables are near each other, the value of this function is small; on the contrary, the two design variables are far away, the value is big. by:

Based on the above equations, the proposed ISC is given

$$
\boldsymbol{p}_{\boldsymbol{i}}=\operatorname{argmax}_{p \in D} \sum_{j=1}^{n} w_{i, j}\left(I\left(\boldsymbol{p}_{i}\right)-I\left(\left(\boldsymbol{p}_{j}\right)\right)\right.
$$

The proposed ISC has the following advantages: 1) it can intelligently add sample points to improve the ANNs, so it can learn with a small number of expensive simulations; 2) it can avoid searching the areas with relative large function values and decrease the computational cost; 3 ) it considers both the similarity of the design variables and the points of high estimated approximation error which generated by ANN model; 4) It is simple and easy to use.

\section{EXPERIMENTAL RESULTS AND DISCUSSION}

In this section, we employed four widely used analytical benchmark problems [14], namely Rosenbrock, Sphere, Six Hump Camel Back functions of 2-dimensional (2D), and 4D Sphere function.

The benchmark functions are chosen to cover a large variety of problem properties. Rosenbrock (2D) and Sphere (2D) functions are unimodal functions. The valley of the global minimum is easy to find, however fine convergence to the global minimum is difficult. Six Hump Camel Back (2D) is a multimodal function. Also, the simulation is conducted on the 4D Sphere function to account for multiple parameters.

We use LHS to generate the initial sample in all cases. In some cases, by pure luck, this initial sample may include a point close to the global optimum, accelerating the search. To avoid any bias when testing, here ANN and ANN with ISC use the same initial sample. In our setup, the simulation and training of the ANN have been performed using the Neuroet toolbox [15]. Furthermore, the transfer function between the input layer and the hidden layer is "log-sigmoide", while the transfer function between the hidden layer and the output layer is "pure-linear". A genetic algorithm is used for both objective-function of interest and for the maximization of ISC.

The performance of the infill criteria can be assessed in a number of ways. Here this performance is measured by the distance of the best finding optimum to the global optimum. To be more precise, for benchmark functions, the true optimum $x^{*}$ is known, an intuitive method of comparison is to find the absolute error between the true optimum $x^{*}$, and best feasible point $x^{\text {best }}$. The absolute error is defined as:

$$
\left\|x^{*}-x^{b e s t}\right\|=\sqrt{\left(x^{*}-x^{b e s t}\right)^{2}} .
$$

A series of 20 runs was performed for each of the 4 test functions. This was done to see at what low evaluation number each method approached convergence for all 20 runs. With that setup, the global optima of the criteria are found in 
the vast majority of the cases for all considered configurations.

\section{A. Sphere Function (2D)}

The first example is the minimization of the Sphere test function, a common and simple benchmark test function used in surrogate-based global optimization. It is given as $f(x)=x_{1}^{2}+x_{2}^{2}$. Test area is usually restricted to hyphercube $-5.12 \leq x_{i} \leq 5.12, i=1,2$. It is continuous, convex and unimodal. The Fig. 3 shows its two-dimensional form. There is a global optima of the Sphere function, for which $f\left(x^{*}\right)=0, x^{*}=(0,0)$.

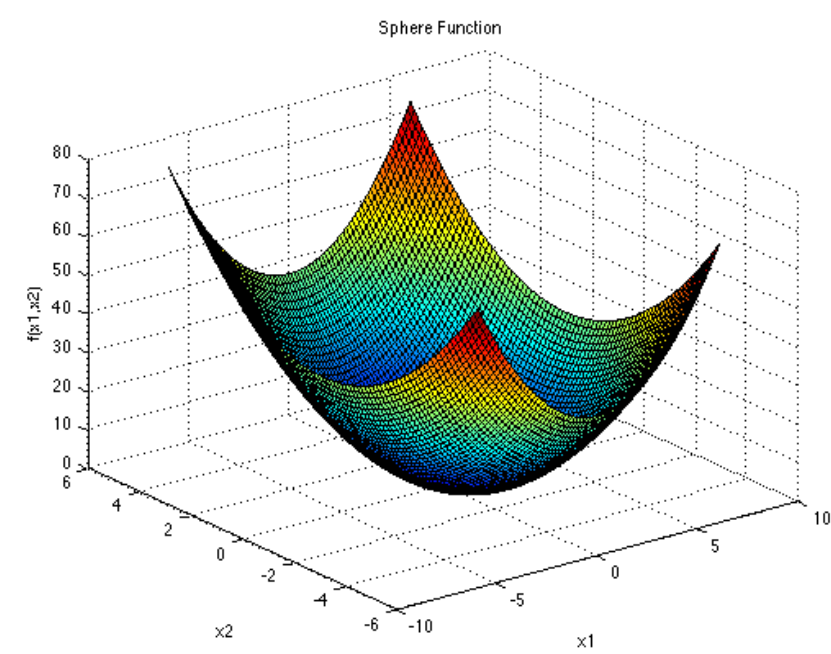

Fig. 3. Sphere function in $2 \mathrm{D}$.

TABLE I: COMPARISON OF ERRORS FOR SPHERE FUNCTION (2D)

\begin{tabular}{cccc}
\hline \hline Method & $\begin{array}{c}\text { The number } \\
\text { of sample } \\
\text { points } m\end{array}$ & $\begin{array}{c}\text { The values of the finding } \\
\text { optima }\end{array}$ & Average error \\
ANN & 10 & $(-1.127472,0.324886)$ & 1.376740 \\
ANN & 30 & $(6.36508 \mathrm{E}-5,-0.008073)$ & $6.51796 \mathrm{E}-05$ \\
ANN & 35 & $(-0.0094507,-0.005342)$ & $1.17858 \mathrm{E}-04$ \\
ANN & 40 & $(-0.0072253,-0.003513)$ & $6.45475 \mathrm{E}-05$ \\
$\begin{array}{c}\text { ANN } \\
\text { with ISC } \\
\text { ANN }\end{array}$ & 11 & $(-0.465341,-0.217933)$ & 0.264037 \\
$\begin{array}{c}\text { with ISC } \\
\text { ANN } \\
\text { with ISC } \\
\text { ANN }\end{array}$ & 15 & $(0.098401,-0.064587)$ & 0.013854 \\
with ISC & 18 & $(-0.015163,0.009281)$ & $3.16055 \mathrm{E}-04$ \\
\hline \hline
\end{tabular}

We select an initial design with $m=10$ points. Table I shows the results of both ANN and ANN with ISC approaches. It can be seen that as $\mathrm{m}$ increases, our model generally becomes more accurate (the error reduces), as expected. Here we compare the accuracy of the optimum of the true function with and without ISC. Both approaches succeed in finding the true optimum. Without ISC the ANN required around 40 data points to reach the global optimum. On the other hand, utilizing the proposed ISC the optimum was found with only 18 points after 8 iterations. So in this case, ANN with ISC is far superior.

\section{B. Six-Hump Camel Back Function (2D)}

Six-Hump Camel back function (2D) is defined as:

$$
f(x)=4 x_{1}^{2}-2.1 x_{1}^{4}+\frac{x_{1}^{6}}{3}+x_{1} x_{2}-4 x_{2}^{2}+4 x_{2}^{4} .
$$

Test area is usually restricted to $-5 \leq x_{i} \leq 5, i=1,2$.
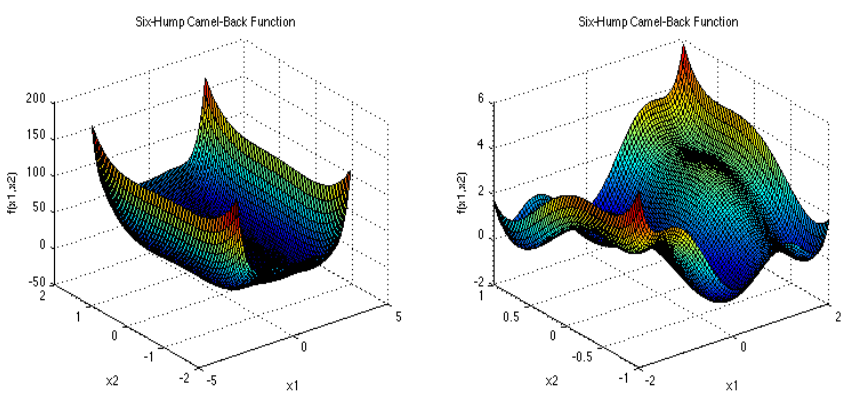

Fig. 4. Six Hump Camel Back Function in different range.

The left plot in Fig. 4 shows the six-hump Camel function on its recommended input domain. The plot on the right shows only a portion of this domain, to allow for easier viewing of the function's key characteristics.

In the continuous domain, six local minima are located, two of them are global minima; namely, $x^{*}=(0.089842$, $-0.712656)$ and $x^{* *}=(-0.089842,0.712656)$ with $f\left(x^{*}\right)=f\left(x^{* *}\right)=-1.031628$. It also has two additional local minima.

We select an initial design with $m=30$ points. Table II shows the results of both ANN and ANN with ISC approaches. Since this test function is complicated, the results of both methods are not entirely accurate. In some cases, the optimization method gets trapped in one of the local minima. Still, the use of the ISC took nearly 36 points for an acceptable solution. Without the ISE, a similar solution was found after 45 points.

TABLE II: COMPARISON OF ERRORS FOR SIX-HUMP CAMEL BACK

\begin{tabular}{cccc} 
Method & $\begin{array}{c}\text { The number } \\
\text { of sample } \\
\text { points } m\end{array}$ & $\begin{array}{c}\text { The values of the finding } \\
\text { optima }\end{array}$ & Average error \\
ANN & 30 & $(1.833207,0.269188)$ & 1.395452 \\
ANN & 35 & $(0.989603,1.410874)$ & 0.909037 \\
ANN & 40 & $(0.340639,0.130975)$ & 0.511647 \\
ANN & 45 & $(-0.813648,0.218690)$ & 0.619639 \\
$\begin{array}{c}\text { ANN } \\
\text { with ISC }\end{array}$ & 31 & $(1.840641,0.107743)$ & 1.367189 \\
$\begin{array}{c}\text { ANN } \\
\text { with ISC } \\
\text { ANN }\end{array}$ & 35 & $(0.0954703,0.004843)$ & 0.507324 \\
with ISC & 36 & $(0.270422,-0.05306)$ & 0.483538 \\
$\begin{array}{c}\text { ANN } \\
\text { with ISC }\end{array}$ & 38 & $(0.409892,0.328426)$ & 0.445692 \\
\hline
\end{tabular}

\section{Rosenbrock Function (2D)}

The Rosenbrock's valley is a classic optimization problem, known as banana function or the second function of De Jong. It is naturally nonseparable and is defined as follows: 


$$
f(x)=100 \times\left(x_{2}-x_{1}^{2}\right)+\left(1-x_{1}\right)^{2} .
$$

Test area is usually restricted to $-2.048 \leq x_{i} \leq$ $2.048, i=1,2$.

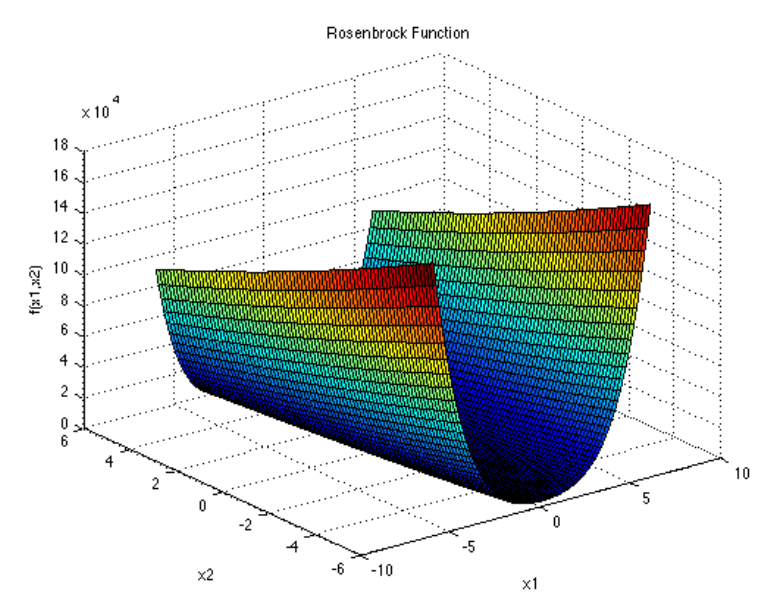

Fig. 5. Rosenbrock function in 2D.

Rosenbrock function (2D) has a global minima $x^{*}=(1,1)$ with $f\left(x^{*}\right)=0$. The global minimum is inside a long, narrow, parabolic shaped flat valley. To find the valley is trivial. To converge to the global minimum, however, is difficult.

Similar to the previous cases, $m=30$ points were selected as initial design and the results are given in Table III. We can see from the table, that the use of the ISC permitted the optimization algorithm to find a solution near to the optimum with a small number of points (36 points). However, we observed very little success at finding optima by ANN with LHS design alone. The optimization algorithm was unable to make any additional progress beyond this point, perhaps struggling to traverse the valley.

TABLE III: COMPARISON OF ERRORS FOR ROSENBROCK FUNCTION (2D)

\begin{tabular}{cccc}
\hline \hline Method & $\begin{array}{c}\text { The number } \\
\text { of sample } \\
\text { points } m\end{array}$ & $\begin{array}{c}\text { The values of the finding } \\
\text { optima }\end{array}$ & Average error \\
ANN & 30 & $(0.326942,0.408716)$ & 0.895891 \\
ANN & 35 & $(-0.10094,0.070638)$ & 1.440765 \\
ANN & 40 & $(0.584282,0.254416)$ & 0.853649 \\
ANN & 45 & $(0.547114,0.299495)$ & 0.834154 \\
ANN & 50 & $(0.593662,0.207915)$ & 0.89023 \\
$\begin{array}{c}\text { ANN } \\
\text { with ISC }\end{array}$ & 31 & $(-0.312919,0.229329)$ & 1.522397 \\
$\begin{array}{c}\text { ANN } \\
\text { with ISC } \\
\text { ANN }\end{array}$ & 35 & $(0.909437,0.954252)$ & 0.101461 \\
$\begin{array}{c}\text { with ISC } \\
\text { ANN } \\
\text { with ISC }\end{array}$ & 36 & $(0.804714,0.947936)$ & 0.202112 \\
\hline \hline
\end{tabular}

\section{Sphere Function (4D)}

The Sphere function (4D) has four parameters. The function is defined as: $f(x)=x_{1}^{2}+x_{2}^{2}+x_{3}^{2}+x_{4}^{2}, \quad-2 \leq$ $x_{i} \leq 2, i=1, \ldots, 4$.
TABLE IV: COMPARISON OF ERRORS FOR SPHERE FUNCTION (4D)

\begin{tabular}{cccc}
\hline \hline Method & $\begin{array}{c}\text { The number } \\
\text { of sample } \\
\text { points } m\end{array}$ & $\begin{array}{c}\text { The values of the finding } \\
\text { optima }\end{array}$ & Average error \\
ANN & 20 & $(1.099,2, \quad 2,1.71)$ & 3.483605 \\
ANN & 40 & $\begin{array}{c}(-0.0537,0.00576, \\
-0.0285,-0.3004)\end{array}$ & 0.306571 \\
& & $(0.03244,-0.01353$, & 0.155925 \\
ANN & 50 & $-0.06338,0.13806)$ & \\
ANN & 55 & $\begin{array}{c}(-0.00164,0.04242, \\
0.09037,0.00972)\end{array}$ & 0.100317 \\
ANN & 21 & $(-0.3707,-1.999$, & 2.879635 \\
with ISC & & $-2,0.3936)$ & 0.273351 \\
ANN & 23 & $(-0.1786,-0.183$, & \\
with ISC & & $-0.0633,0.0728)$ & 0.264952 \\
ANN & 26 & $(-0.0093,-0.1436$, & \\
with ISC & 26 & $-0.1662,-0.1478)$ & \\
ANN & \multirow{2}{*}{28} & $(-0.0085,-0.09655$, & \\
with ISC & & $-0.06465,-0.1295)$ & \\
\hline \hline
\end{tabular}

From Table IV, the results in 4D sphere function are similar to $2 \mathrm{D}$ case. Both approaches succeed in finding the true optimum. However, less sample points are required if the ISC guides the selection of the design points to be sampled.

To summarize the results, boxplots for the sphere 4D, six-hump carmel, and Rosenbrock function are presented in Fig. 6 and Fig. 7 which are more visualized. Fig. 6 presents the results with ISC and the Fig. 7 without ISC method. It turns out that use of ISC has a superior performance on all benchmark functions.

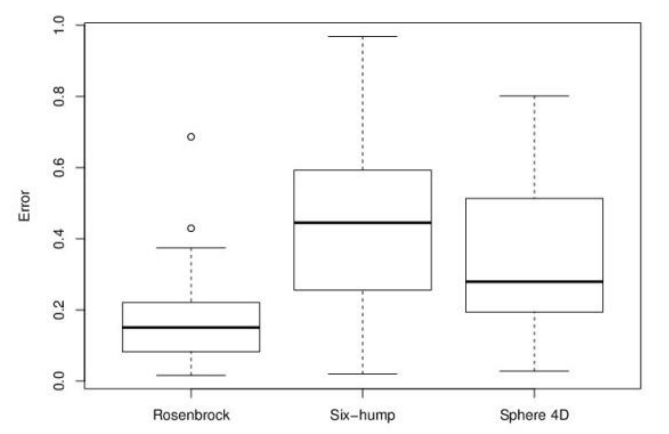

Fig. 6. Boxplot of the errors by ANN with ISC method over 20 runs.

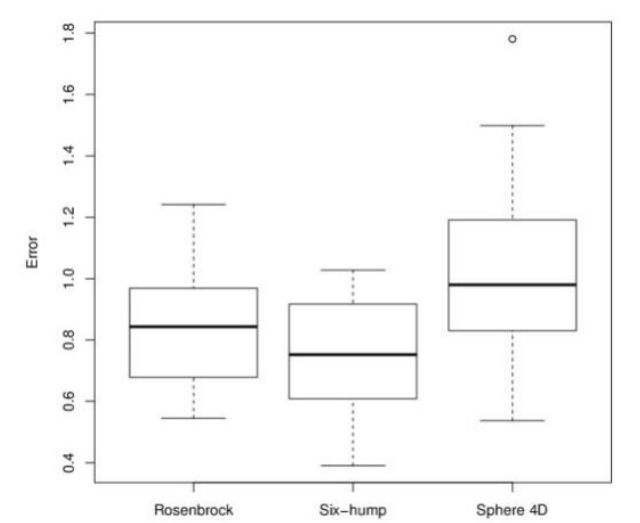

Fig. 7. Boxplot of the errors by ANN method over 20 runs.

\section{CONCLUSION AND Future WORK}

In this paper, an optimization method using ANN with ISC is developed. A predictive model including design 
parameters is built by using ANN with ISC to reduce the computational time. By comparing ANN and ANN with ISC methods based on a benchmark with a variety of test functions, we get the results showing that the proposed method can identify and calculate design parameters with a minimum number of computer simulations.

It must be mentioned that the proposed ISC assumes that the design space is fixed, and the range of variables is based only on the criteria of the experienced researchers in their related fields. Further to this, by selecting model update points is close proximity to the constraint boundaries, the regions that are likely to contain the feasible optimum can be better modeled. Thus, a changeable design space will be taken into consideration in research.

\section{ACKNOWLEDGMENT}

The work carried out in this paper was supported by a grant from the Japan Society for the Promotion of Science under Grant-in-Aid for Scientific Research S No. 24226015.

I gratefully acknowledge helpful discussions with Prof. Shimizu and Prof. Sakaguchi.

\section{REFERENCES}

[1] M. Dao, N. Chollacoop, K. J. V. Vliet, T. A. Venkatesh, and S. Suresh, "Computational modeling of the forward and reverse problems in instrumented sharp indentation, " Acta Materialia, vol. 49, pp. 3899 3918, 2001.

[2] J. L. Bucaille, S. Stauss, E. Felder, and J. Michler, "Determination of plastic properties of metals by instrumented indentation using different sharp indenters," Acta Materialia,, vol. 51, pp. 1663-1678, 2003.

[3] H. Toda, S Masuda., R. Batres, M. Kobayashi, S. Aoyama, and M. Onodera et al., "Statistical assessment of fatigue crack initiation from sub-surface hydrogen micropores in high-quality die-cast aluminum," Acta Materialia, vol. 59, pp. 4990-4998, 2011.

[4] A. I. Khuria and M. Conlona., "Simultaneous optimization of multiple responses represented by polynomial regression functions," Technometrics, vol. 23, pp. 363-375, 1981.

[5] A. J. S. Technometrics and J. Q. Broughton, "Neural networks in computational materials science: training algorithms," Model. Simul. Mater. Sc., vol. 3, pp. 371-390, 1995.

[6] A. Cichocki and R. Unbehaven, Neural Networks for Optimization and Signal Processing, 1st ed. Chichester, U.K.: Wiley, 1993, vol. 2, pp. 45-47.

[7] N. Dyn, D. Levin, and S. Rippa, "Numerical procedures for surface fitting of scattered data by radial basis functions", SIAM Journal of Scientific and Statistical Computing, vol. 7, no. 2, pp. 639-659, 1986.

[8] J. B. Sacks and W. J. Welch, "Designs for computer experiments," Technometrics, vol. 31, no. 1, pp. 41-47, 1989.

[9] J. B. Sacks, W. J. Welch, T. J. Mitchell, and H. P. Wynn, "Design and analysis of computer experiments," Statistical Science, vol. 4, no. 4, pp. 409-435, 1989

[10] D. R. Jones, M. Schonlau, and W. J. Welch, "Efficient global optimization of expensive black-box function," Journal of Global Optimization, vol. 13, pp. 455-492, 1998.

[11] F. A. C. Viana and R. T. Hafika, "Surrogate-Based optimization with parallel simulations using the probability of improvement," AIAA, pp. 2010-9392, 2010.

[12] M. D. Mackay, R. J. Beckman, and W. J. Conover, "A comparison of three methods for selecting values of input variables in the analysis of output from a computer code", Technometrics, vol. 21, pp. 239-245, 1979.

[13] D. Bursztyn and D. M. Steinberg, "Comparison of designs for computer experiments," Journal of Statistical Planning and Inference, vol. 136, pp. 1103-1119, 2006.

[14] L. Dixon and G. Szego, "Towards global optimization 2," Amsterdam, vol. 2, ch. 5, North Holland, 1978.
[15] A. P. Noble and H. E. Tribou, "Neuroet: An easy-to-use artificia neural network for ecological and biological modeling," Ecological Modeling, vol. 203, pp. 87-98, 2007.

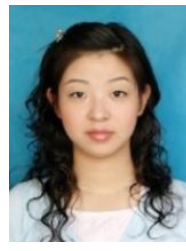

Han Li received BS degree in mathematics and applied mathematics from Faculty of Mathematics and Compute Science in Hubei University in 2007. She received her $\mathrm{Ph} . \mathrm{D}$. degree in the School of Mathematics and Statistics at Beijing University of Aeronautics and Astronautics. Her research interests include neural networks, learning theory and pattern recognition. She now works as a researcher in the Department of Mechanical Engineering, Toyohashi University of Technology. Dr. Li belongs to the Industrial Systems Engineering Group in Toyohashi University of Technology.

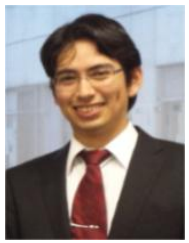

Leonardo Gutierrez received his BS in industria engineering from the National Polytechnic Institute in 2012. Currently, Leonardo is pursuing his master's degree in mechanical engineering at Toyohashi University of Technology in Japan. He worked as Jr. Functional Oracle E-Business Suite Consultant for SIR Tecnologia S.A., outsourced in Grupo Aeromexico S.A., Oracle Mexico S.A., Grupo GEO S.A. and The Solocup Company Mexico S.A. until 2011. Current research fields include Industrial Engineering and Optimization Engineering. Mr. Gutierrez belongs to the Industrial Systems Engineering Group in Toyohashi University of Technology

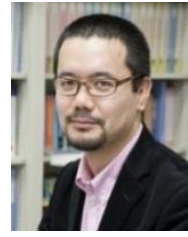

Osamu Kuwazuru received his bachelor of engineering from Faculty of Engineering, Shibaura Institute of Technology in 1996, his master of engineering degree in 1998 and doctor of engineering degree in 2001 from the University of Tokyo. His research interests include computational solid mechanics, nonlinear numerical modeling, 4D imaging, fatigue, corrosion, and micromechanics. He worked as an assistant professor at Institute of Industrial Science, the University of Tokyo between 2001 and 2008 , and currently works as an associate professor at University of Fukui since 2008. Dr. Kuwazuru belongs to Department of Nuclear Power \& Energy Safety Engineering, University of Fukui.

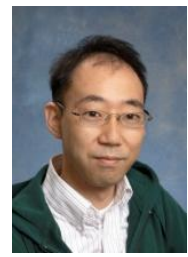

Masakazu Kobayashi received his bachelor of engineering degree from the Faculty of Engineering, Utsunomiya University in 1996, his master of engineering degree in 1998 and his doctor of engineering degree in 2001 also from Utsunomiya University, Japan. His major is development of $3 \mathrm{D}$ analysis method for material microstructure and its application. He worked as a researcher at Utsunomiya University before moving on to Toyohashi. He currently works as an associate professor at the Department of Mechanical Engineering, Toyohashi University of Technology.

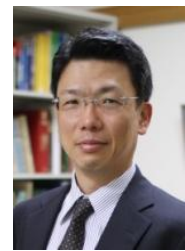

Hiroyuki Toda graduated from Kyoto University in 1987, and received his doctor of engineering degree in 1995 . He is currently a distinguished professor in structural materials and a director of the 3D/4D Structural Materials Research Centre in Kyushu University. His research interests are 3D/4D image-based analysis and its application to understand microstructure-properties relationships in engineering material.

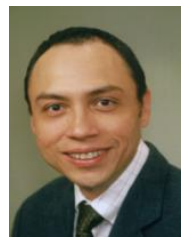

Rafael Batres is an associate professor of industrial and systems engineering in the Mechanical Engineering Department at Toyohashi University of Technology. He received a BS in chemical engineering from the National University of Mexico (UNAM) and both a MEng and PhD in process systems engineering from Tokyo Institute of Technology. His research is currently focused on material and product design, design and simulation of sustainable systems, systematic methods for ontology development, and large-scale data management and analytics. 